\title{
Curvature and Velocity of Methane-Air Bunsen Flame Tips
}

\author{
G. García-Soriano • P. L. García-Ybarra • \\ F. J. Higuera
}

\begin{abstract}
PIV and photographic recording are used to measure the velocity of the fresh gas and the shape of the reaction layer in a region around the tip of a methaneair Bunsen flame attached to a cylindrical burner. The results compare well with numerical simulations carried out with an infinite activation energy reaction model. The experimental and numerical results confirm that the well-known linear relation between flame velocity and flame stretch derived from asymptotic theory for weakly curved and strained flames is valid for small and moderate values of the flame stretch if the modified definition of stretch introduced by Echekki and Mungal (Proc Combust Inst 23:455-461, 1990) and Poinsot et al. (Combust Sci Technol 81:45-73, 1992 ) is used. However, the relation between flame velocity and modified stretch ceases to be linear and approaches a square root law for large values of the stretch, when the curvature of the flame tip becomes large compared to the inverse of the thickness of a planar flame.
\end{abstract}

Keywords Combustion • PIV • Flame aerodynamics

\section{Introduction}

The convection-diffusion balance in the transport region of a premixed flame is affected by the curvature of the flame and the nonuniformity of the flow. Analysis [1-3] shows that, in certain conditions, there is a linear relation between the so-called stretch of the flame, which is the logarithmic derivative of the area $\delta A$ of an element of a propagating flame front, and the change of its local normal velocity relative to

G. García-Soriano · P. L. García-Ybarra

Departamento de Física Matemática y de Fluidos, UNED, Madrid, Spain

F. J. Higuera (困)

E. T. S. Ingenieros Aeronáuticos, UPM, Madrid, Spain

e-mail: fhiguera@aero.upm.es 
the fresh gas, $U_{n}$, about the velocity of the planar flame, $U_{L}$. The Markstein length $\mathcal{L}$ is the proportionality constant in this linear relation:

$$
U_{n}-U_{L}=-\mathcal{L} \frac{1}{\delta A} \frac{\mathrm{d} \delta A}{\mathrm{~d} t} .
$$

For a stationary flame, the stretch of an element of the flame is the sum of two contributions,

$$
-\frac{1}{\delta A} \frac{\mathrm{d} \delta A}{\mathrm{~d} t}=U_{L} \mathcal{C}+\boldsymbol{n} \cdot \nabla \boldsymbol{v} \cdot \boldsymbol{n},
$$

where the first term on the right-hand side is the effect of the front curvature for a flame moving with a normal velocity $U_{L}$ and the second term is the rate of strain of a material surface in the shape of the flame immersed in the velocity field $\boldsymbol{v}$ of the fresh gas upstream of the flame. Here $\boldsymbol{n}$ is a unit vector normal to the flame pointing toward the burnt gas and $\mathcal{C}=\nabla \cdot \boldsymbol{n}$ is twice the mean curvature of the front.

These results have been rigorously derived using high activation energy asymptotics in the limit of infinitely thin flames; i.e., when the curvature of the flame is small compared to the inverse of its thickness and the strain rate of the flow is small compared to the inverse of the transit time of the gas across the flame [1-3]. The theory also provides an expression for the Markstein length in terms of the physicochemical properties of the gas. However, the question arises as to what extent can these results be used for real flames of finite thickness, for which even the definitions of propagation velocity and front curvature are ambiguous. For planar stationary flames in the stagnation point flow, the question has been addressed by Davis et al. $[4,5]$, who used numerical computations to show that Eqs. 1 and 2 can still be used if the velocity $U_{n}$ and the strain rate are understood as those of the cold gas upstream of the flame (the outer solution in the asymptotic description) extrapolated to the reaction region of the flame. On the other hand, on the basis of their experimental and numerical results for two-dimensional Bunsen flames, Echekki and Mungal [6] and Poinsot et al. [7] proposed that Eqs. 1 and 2 are valid for strongly curved flames if the curvature of the thin reaction region is used for $\mathcal{C}$ in Eq. 2 and the velocity $U_{L}$ in this equation is replaced by the velocity $U_{n}$ of the curved and strained flame. For a general stationary flame, this should be the velocity of the cold gas extrapolated to the reaction layer, but such extrapolation was not needed in the approximately uniform cold gas flow of $[6,7]$.

The value of the Markstein length can be obtained from Eqs. 1 and 2 if the local flame velocity and the flame and flow geometry can be determined experimentally. The experimental task can be simplified if the experiment is designed in such a way that only one of the two contributions to the flame stretch discussed above is present or there is a relation between the two. This goal has been achieved in previous works by using spherical flames propagating inward or outward [8], or flames in the stagnation point flow configuration. In the first case the front curvature alone determines the stretch, whereas in the second case the flame is flat and the only contribution comes from the strain rate [9].

A circular jet burner leading to a Bunsen flame has been chosen in the present work to further investigate the validity of the extended form of Eqs. 1 and 2. This configuration was considered before by Mungal et al. [10]. It allows to simultaneously measure the planar flame propagation velocity, the local flame propagation velocity (defined as in [4-7]), and the curvature of the flame tip. In addition, strongly curved 
flames can be obtained by increasing the ratio $U_{0} / U_{L}$ of the gas injection velocity to the velocity of the planar flame. This allows further checking the validity of the results obtained in $[6,7,10]$.

\section{Methods}

\subsection{Experimental setup and techniques}

A methane-air mixture is formed in a plenum chamber from separate streams of these gases, whose flow rates are measured with Bronkhost mass flow controllers. The mixture is fed to a vertical circular tube which is fitted with several honeycombs and flow uniformizing grids and ends in a convergent nozzle $2 \mathrm{~cm}$ in diameter, where a flame is attached.

The temperature of the cold gas is measured with a thermocouple at the nozzle exit. The velocity field of the fresh gas in a vertical plane through the axis of the burner is measured using a PIV system composed of a $532 \mathrm{~nm} \mathrm{Nd:YAG} \mathrm{laser} \mathrm{(New}$ Wave 120XT) with sheet-forming emission optics, a 1,392 × 1,040 pixels CCD camera (PCO Pixel Fly) with a Nikon automatic macro lens, and a pulse and delay generator (ILA). The camera is set perpendicular to the laser sheet, imaging a region of about $30 \times 22 \mathrm{~mm}$, which gives a resolution of 46 pixe $1 / \mathrm{mm}$, or about 33 pixels across the preheating region of a typical flame. Inter-pulse times are of the order of $70 \mu \mathrm{s}$. Cross-correlation computations are done with the ViDPIV software, which uses cascades of interrogation windows starting with sizes of about $32 \times 32$ pixels. The gas is seeded with olive oil drops formed by evaporation-condensation of a 100:1 oil-water emulsion in a seeding chamber inserted in the air line. These oil drops evaporate in the preheating region of the flame, which therefore appears dark in the images. It is not possible to measure the velocity of the gas in and behind the flame using oil drops. However, this does not pose a problem here because only the velocity of the cold gas ahead of the flame appears in the theory leading to Eqs. 1 and 2 ; see [1-5]. Comparisons with flames in unseeded mixtures show that the additional fuel in the oil drops has no noticeable effect on the flame.

As was pointed out by Mungal et al. [10], the radiation emitted by the reaction layer of the flame in a narrow band around the laser frequency goes through the filter set in front of the camera and forms an outline of this layer in the second, long exposure picture of each PIV couple. Even though this radiation comes from the reaction region of the whole flame, it is dominated by the contribution of the section where the camera is focused and has been used to define the shape of this section of the reaction layer.

Figure 1a is a raw sample (negative) picture showing the light scattered by the oil drops in the cold gas, the light emitted by the luminous region of the flame, and the dark preheating region depleted of drops in between. Figure $1 \mathrm{~b}$ shows the modulus of the cold gas velocity measured with PIV. As can be seen, this velocity is nearly uniform at the outlet of the nozzle and stays uniform in horizontal planes, increasing with height above the nozzle. Assuming that the flow of the cold gas is axisymmetric, the strain rate tensor is a diagonal tensor on the symmetry axis and depends only on the axial derivative of the axial component of the velocity. This derivative is computed from the measured velocity. Since the derivative is nearly uniform across 

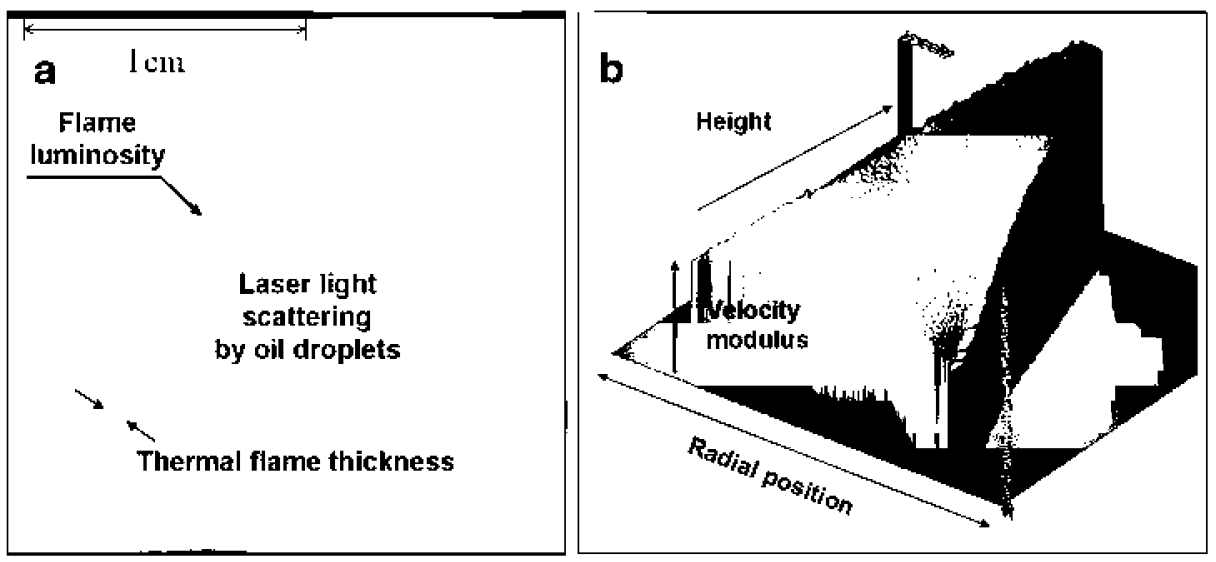

Fig. 1 a Negative picture of the light scattered by the oil drops in the cold gas and the light emitted by the reaction region of the flame, with the preheating region depleted of drops in between. b PIV map of the velocity upstream of the flame, showing the plug flow at the outlet of the burner and the increase of the gas velocity with height

the cold gas, the error of this computation is not expected to be much larger than the error of the measured velocity itself.

The value obtained extrapolating the velocity of the cold gas along the axis of the burner to the tip of the luminous layer is denoted $u_{t}$ hereafter. This velocity plays at the tip of the flame the role of $U_{n}$ in the general discussion of the previous section. The velocity of the planar flame is determined by measuring $u_{t}$ for different injection velocities $\left(U_{0}\right)$ and extrapolating the results to the limit of zero curvature.

\subsection{Numerical techniques}

The flow and the temperature distribution around the tip of a stationary axisymmetric flame are computed numerically assuming that combustion occurs through a single irreversible Arrhenius reaction with infinitely high activation energy. The reaction layer is then an infinitely thin free boundary where well-known jump relations must be satisfied [11]. The Lewis number of the reactant that is depleted by the flame is assumed to be equal to the unity, which is a reasonable approximation for methane-air flames. In these conditions, the final combustion temperature and the burning rate are independent of the curvature of the flame and the strain rate of the flow. The mass, momentum, and energy conservation equations are solved together with the equation of state in the quasi-isobaric low Mach number approximation. The conditions for the temperature at the reaction sheet are (see, e.g., [11]) $T=T_{b}$ and $\lambda_{b} \partial T /\left.\partial n\right|_{0^{-}}=\rho_{u} U_{L} c_{p}\left(T_{b}-T_{u}\right)$, where $\rho_{u}$ and $T_{u}$ are the density and temperature of the cold gas, $T_{b}$ is the adiabatic flame temperature, $\lambda_{b}$ is the thermal conductivity of the gas at temperature $T_{b}, c_{p}$ is the specific heat at constant pressure, which is assumed to be constant, and $n$ is the distance to the reaction sheet. A formulation of the mathematical problem can be found in [12].

When the injection velocity of the fresh gas, $U_{0}$, is large compared to the velocity of the planar flame $U_{L}$, the flame approaches a slender cone of semiangle $U_{L} / U_{0}$ everywhere except in a region around the apparent vertex of the cone which is small 
compared to the radius of the nozzle. The conservation equations can be solved in this region using approximate far field boundary conditions that express the matching with the larger region where the flame is conical. With these approximate conditions, the flow of the fresh gas at the inlet boundary of the computational domain is taken to be a uniform vertical stream with temperature $T_{u}$ and the flow of the burnt gas far downstream of the flame approaches the superposition of a uniform vertical stream plus a distribution of sources along the axis of the flame with a strength proportional to the distance to its apparent tip; see $[12,13]$ for details. The temperature of the gas is $T=T_{b}$ downstream of the reaction sheet.

\section{Results and Discussion}

\subsection{Flow and flame tip shape}

The shape of the luminous layer of the flame and the velocity field of the cold gas were determined for different values of the injection velocity, and therefore of the height of the flame, keeping the equivalence ratio $\phi$ constant. Rich flames $(\phi>1)$ were used because the diffusion flame of the excess fuel with ambient oxygen which appears then downstream of the primary premixed flame was found to cause an upward flow that helps stabilizing the tip of the premixed flame. The thick solid curve in Fig. 2a shows the shape of the luminous layer extracted from the photographs

Fig. 2 a Shape of the reaction sheet for $\gamma=5$ and $u_{t} / U_{L}=4.15$. Experimental results (solid) and numerical results (dashed) are included. Also plotted are ten equispaced isotherms between $T=T_{u}$ and $T=T_{b}$ (thin solid). b Dimensionless curvature of the reaction sheet in the upper panel; experimental results (solid) and numerical results (dashed). Here $x$ is the vertical distance from the tip of the reaction sheet and $r$ is the horizontal distance from the axis of the burner
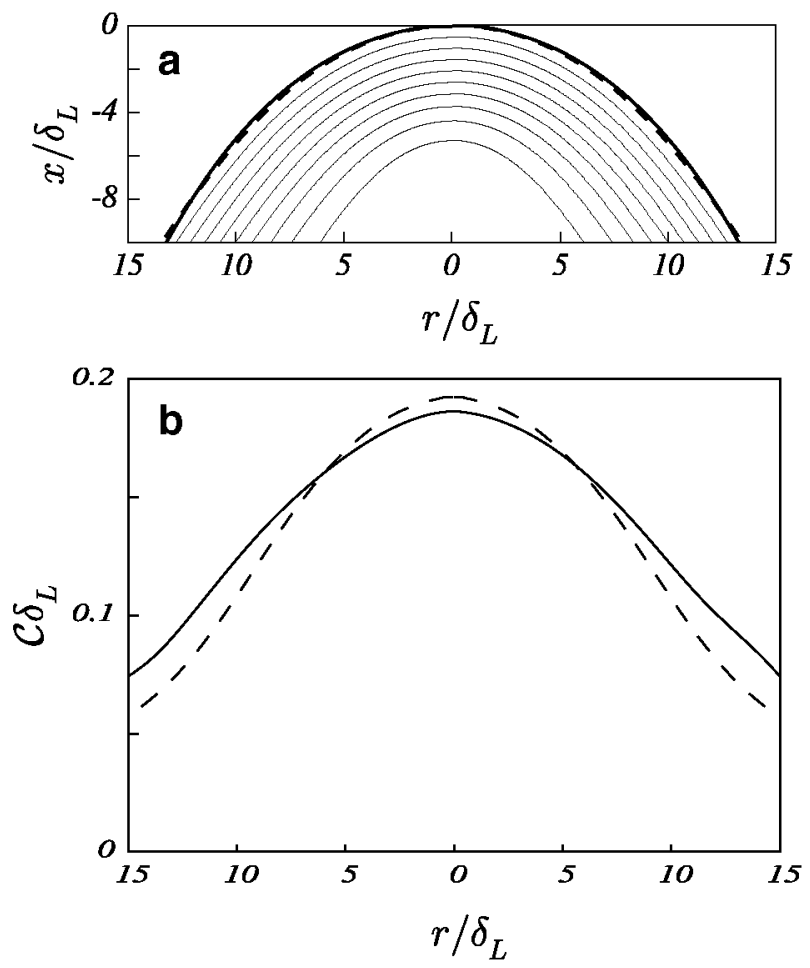
for a sample case with $\phi=1.49$ (for which $\left.\gamma=\left(T_{b}-T_{u}\right) / T_{u} \approx 5\right)$ and $u_{t} \approx 4.15 U_{L}$. The solid curve in Fig. $2 \mathrm{~b}$ shows the curvature of the luminous layer in Fig. 2a. The temperature of the cold gas was $T_{u}=315 \mathrm{~K}$ and the velocity of the planar flame was $U_{L} \approx 14.2 \mathrm{~cm} / \mathrm{s}$. Distances in these figures are scaled with the reference flame thickness $\delta_{L}=\alpha_{u} / U_{L}$, where $\alpha_{u}=0.226 \mathrm{~cm}^{2} / \mathrm{s}$ is the thermal diffusivity of the gas at temperature $T_{u}$. The figures compare the experimental shape and curvature of the reaction sheet with those computed numerically for the same values of $\gamma$ and $u_{t} / U_{L}$ (dashed curves). The thin solid curves in Fig. 2a are computed isotherms. As can be seen, the agreement between experimental and numerical results is fairly good, which is somewhat unexpected given the drastic simplification of the reaction kinetics used in the numerical computations. The discrepancies in Fig. 2b can be attributed partially to the spline algorithm used to differentiate the digitized contour in the photographs, which tends to smooth the contour and decrease its curvature, and partially to the numerical errors incurred by the simplification of the kinetics and by setting the far field boundary conditions at a finite distance from the symmetry axis, equal to $30 \delta_{L}$ in the present computations. This seems to artificially confine the flow, restricting somewhat its lateral expansion and increasing the curvature of the reaction sheet at the tip.

Figure 3 shows the dimensionless distributions of gas velocity and temperature along the symmetry axis computed for $\gamma=5$ and three values of the ratio $u_{t} / U_{L}$. This figure and Fig. $1 \mathrm{~b}$ show that the velocity of the cold gas increases streamwise before reaching the preheating region of the flame where the temperature rises, a result also apparent in Fig. 8 of [10]. This is an important difference with two-dimensional Bunsen flames, for which the velocity of the cold gas is nearly uniform $[6,7,14]$. The difference can be traced to the pressure distribution in the burnt gas downstream of a conical flame, which is felt by the cold gas because the pressure jump across the flame is a constant equal to $\gamma \rho_{u} U_{L}^{2}$ in the region of constant flame propagation velocity. Due to the radial divergence of the burnt gas, the pressure that this gas exerts on the flame decreases on approaching the apparent vertex of the cone (see

Fig. 3 Numerical distributions of velocity (solid curves, left-hand side scale) and temperature (dashed curves, right-hand side scale) along the symmetry axis for $\gamma=5$ and $u_{t} / U_{L}=5.24,8.35$, and 11.6, increasing from bottom to top. The dotted line illustrates the extrapolation of the cold gas velocity to the reaction region (here $x=0$ ) used to compute $u_{t}$

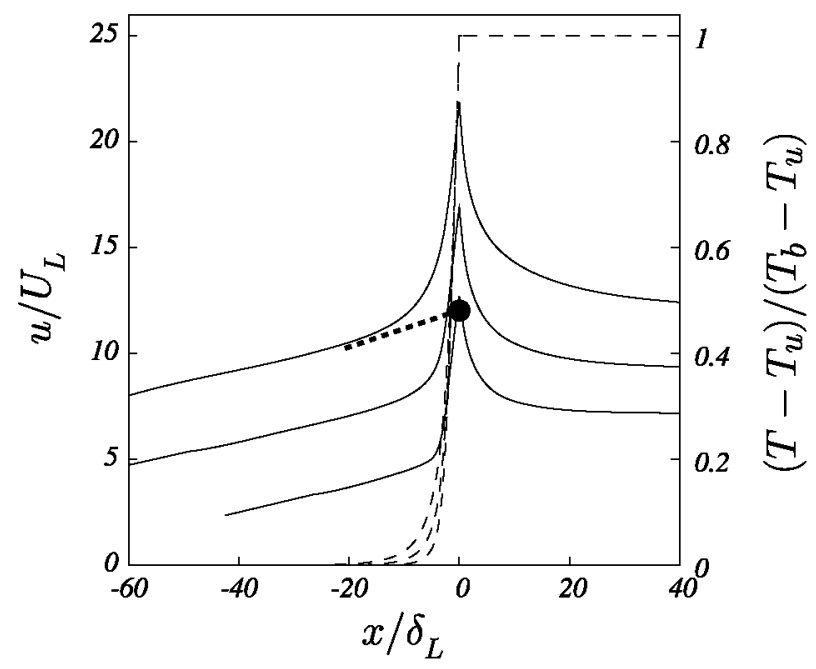


[13] for details), which causes the vertical acceleration of the cold gas. The dotted line in Fig. 3 illustrates how $u_{t}$ is determined from a given velocity profile.

\subsection{Extended Markstein relation}

At the tip of a weakly curved Bunsen flame, Eqs. 1 and 2 give

$$
\frac{u_{t}}{U_{L}}-1=M a\left[\delta_{L} \nabla \cdot \boldsymbol{n}+\frac{\partial\left(u / U_{L}\right)}{\partial\left(x / \delta_{L}\right)}\right],
$$

where $x$ and $u$ are the distance and gas velocity along the symmetry axis, the derivative $\partial u / \partial x$ is evaluated immediately upstream of the flame, and $M a=\mathcal{L} / \delta_{L}$ is the Markstein number. Asymptotic analysis for a single overall Arrhenius reaction with infinitely high activation energy in a mixture with $L e=1$ and a thermal conductivity of the form $\lambda(T)=\lambda_{u}\left(T / T_{u}\right)^{\kappa}$ yields the theoretical Markstein number $M a=(1+\gamma)\left[(1+\gamma)^{\kappa}-1\right] / \kappa \gamma ;$ see $[1-3,15]$.

The heuristic proposal of Echekki and Mungal [6] and Poinsot et al. [7] extends Eqs. 1 and 2 to strongly curved and strained stationary flames by replacing $U_{L}$ in Eq. 2 with $U_{n}$ and evaluating this velocity and the strain rate in Eq. 2 as the values of the cold gas velocity and strain rate extrapolated to the reaction sheet. At the tip of a Bunsen flame, this gives the modified relation

$$
\frac{u_{t}}{U_{L}}-1=M a\left[\frac{u_{t}}{U_{L}} \delta_{L} \nabla \cdot \boldsymbol{n}+\frac{\partial\left(u / U_{L}\right)}{\partial\left(x / \delta_{L}\right)}\right]
$$

to replace Eq. 3 .

The relations 3 and 4 have been tested using the experimental and numerical techniques described above. On the experimental side, a series of experiments has been conducted with $\phi=1.43$ and different heights of the flame. The value of the planar flame velocity obtained from extrapolation of the experimental results to zero stretch is $U_{L} \approx 22.2 \mathrm{~cm} / \mathrm{s}$, which is somewhat higher than the accepted value for $\phi=1.43$. The difference is probably due to the heating of the fresh gas stream by the burner in our experiments. The strain rates computed from the PIV velocity fields are nearly uniform in the cold gas, which makes a simple linear extrapolation to the reaction sheet appropriate. Moreover, the effect of this strain rate in Eqs. 3 and 4 is small compared to that of the curvature for all but the smallest values of $u_{t} / U_{L}-1$. On the numerical side, computations have been carried out for different values of $\gamma$ and $u_{t} / U_{L}$ with $\kappa=0.76$.

The left- and right-hand sides of Eq. 3 evaluated with these results are shown in the vertical and horizontal axes of Fig. 4a. The Markstein number $M a=4.6$, which is not far from the theoretical value for $\gamma=5$, is obtained by fitting Eq. 3 to the experimental results for short flames, while the results for the highest flames in the experiments depart from the linear relation (Eq. 3) (note that the dashed line in Fig. 4a has unit slope). The numerical results for different values of $\gamma$ (open symbols) are reduced using the corresponding theoretical Markstein numbers, which successfully collapses them onto a single curve. This curve differs from the dashed line in Fig. 4a because the numerical results are for fairly high flames (large values of $u_{t} / U_{L}$ ). The numerical results clearly suggest a linear relation between flame velocity and flame stretch for large values of the stretch, but this relation differs from the established linear relation for weak stretch (see inset). A qualitative order-of- 

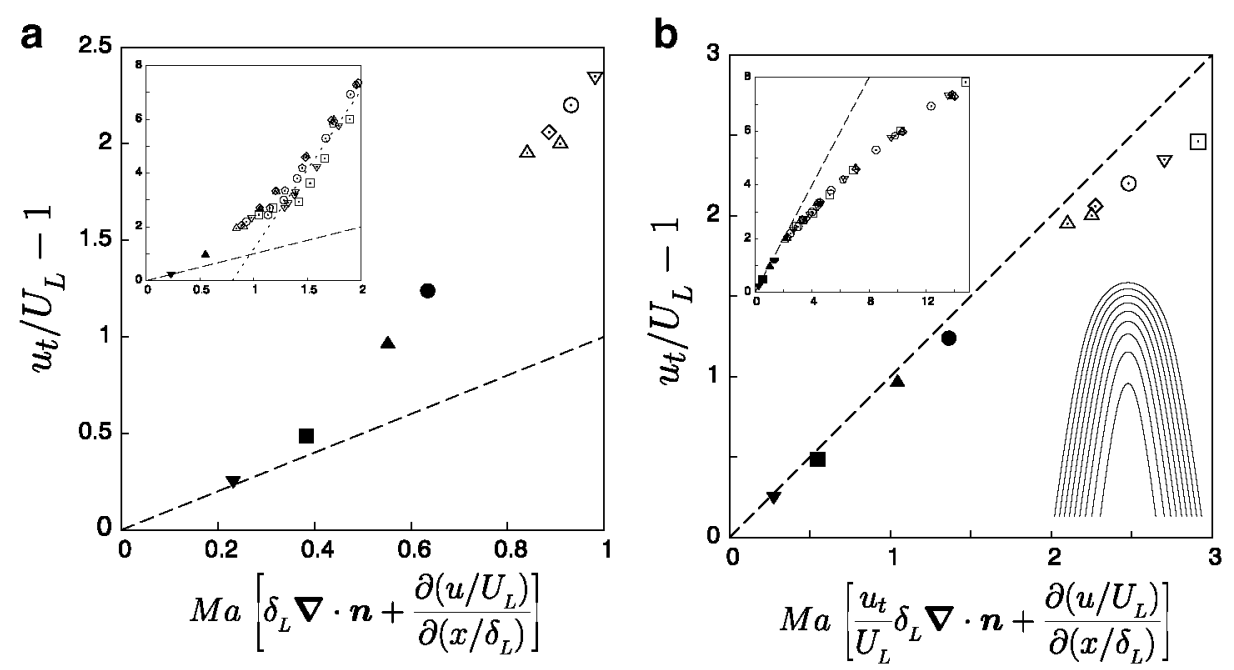

Fig. 4 Left- and right-hand sides of Eqs. 3 (in a) and 4 (in b) evaluated with the experimental data for various flame heights (color, full symbols) and with the numerical data for $\gamma=3(\Delta), 5(0), 6(\nabla)$, and $7(\bullet)$. Dashed lines have unity slope. The inset at the right-hand side of panel $\mathbf{b}$ shows a sample flame tip for a high value of $u_{t} / U_{L}$

magnitude analysis of the region around the tip of a very high flame rationalizes this result; see Section 3.3 .

Figure $4 \mathrm{~b}$ shows the left- and right-hand sides of Eq. 4 evaluated with the same experimental and numerical data as in Fig. 4a. As can be seen, the results stay near the diagonal (dashed line) for a wide range of values of the modified stretch before taking on a square root trend for large values of this variable, which is consistent with the linear trend in Fig. 4a. Figure $4 b$ proves that the modified stretch introduced by Mungal and coworkers [6, 7] is useful as a working correlation, even though Eq. 4 is not a rigorous result and cannot fully account for the experimental and numerical data even in the range of moderate stretch where it is useful.

\subsection{Very high flames}

The radii of curvature of the sections of the reaction sheet in Figs. 1a and 2a are of the order of the thickness of the transport region of the flame. This is shown by the dark region depleted of oil drops in Fig. 1a and by the isotherms in Fig. 2a. However, when the injection velocity and thus the velocity ratio $u_{t} / U_{L}$ increases, the curvature of the tip increases and the rounded cap in these figures tends to become a slender region, in the sense that the increase of the temperature of the gas on the symmetry axis from $T_{u}$ to $T_{b}$ occurs over a distance that begins to be large compared to the radius of a horizontal section of the reaction sheet. A sample tip of this kind is shown in the right-hand side inset of Fig. 4b. Asymptotic analyses for very slender tip regions, for which vertical convection and radial conduction balance each other, have been carried out by Buckmaster [16] in the thermodiffusive limit and by Buckmaster and Crowley [17] taking into account the thermal expansion of the gas.

For large values of the thermal expansion $\gamma$, the characteristic length of the slender tip region where the distance $r_{b}$ from the reaction sheet to the symmetry 
axis is of the order of the thickness of the planar flame, $\delta_{f}$, so that heat conduction from the reaction sheet reaches the symmetry axis, is $\ell_{t}=\delta_{f} u_{t} /\left(\gamma U_{L}\right)$. This estimate follows from the order-of-magnitude balance of the vertical mass flux of reactant in the tip region, where the vertical velocity of the gas is of order $u_{t}$ and its density is of order $\rho_{u} / \gamma$, and the mass flux of reactant consumed in the reaction sheet of a flame whose burning rate is equal to that of the planar flame; i.e., $\left(\rho_{u} / \gamma\right) u_{t} \delta_{f}^{2} \sim \rho_{u} U_{L} \delta_{f} \ell_{t}$. Here $\delta_{f}=\left(\lambda_{b} / \lambda_{u}\right) \delta_{L}$, where $\lambda_{u}$ is the thermal conductivity of the cold gas; see, e.g., [11]. According to this estimate of $\ell_{t}$, the tip region changes from slender to rounded $\left(\ell_{t} \sim \delta_{f}\right)$ when $u_{t}$ decreases to values of order $\gamma U_{L}$; see also [12].

At distances $(-x) \ll \ell_{t}$ from the uppermost point of a slender reaction sheet, where $r_{b} \ll \delta_{f}$, the order-of-magnitude balance of axial convection and radial conduction can be written as $\left(\rho_{u} / \gamma\right) c_{p} u_{t} \Delta T /(-x) \sim \lambda_{b} \Delta T / r_{b}^{2}$, where $\Delta T$ is the order of the spatial variations of the gas temperature. Using the definitions of $\delta_{f}$ and $\ell_{t}$ above, this balance gives the paraboloidal reaction layer $r_{b} / \delta_{f} \sim\left[(-x) / \ell_{t}\right]^{1 / 2}$, whose curvature at the symmetry axis is $\nabla \cdot \boldsymbol{n} \sim \ell_{t} / \delta_{f}^{2}=\left(u_{t} / U_{L}\right) /\left(\gamma \delta_{f}\right) \gg 1 / \delta_{f}$. This amounts to the linear relation

$$
\frac{u_{t}}{U_{L}} \sim \gamma \delta_{f} \nabla \cdot \boldsymbol{n}
$$

between curvature and velocity, which accounts for the numerical results for high flames in Fig. 4.

\section{Conclusions}

The flow and the shape of the reaction layer around the tip of a methane-air Bunsen flame have been measured using PIV and photographic records. The results compare well with numerical simulations carried out with an infinite activation energy reaction. The non-uniform pressure of the burnt gas on a conical flame induces an acceleration of the cold fresh gas ahead of the flame. When the injection velocity of the fresh gas increases, the tip is predicted to evolve from a rounded cap, with a curvature radius of the order of the thickness of the preheating region of the flame, to a slender structure with larger curvature. The numerical and experimental results confirm that the modified definition of flame stretch introduced by Echekki and Mungal and Poinsot et al. allows to extend the linear relation between flame velocity and stretch to non-small values of the stretch. However, the linear relation becomes less accurate when the stretch is very large, and it is predicted to break down when the tip becomes slender.

Acknowledgements This work was supported through projects HYSYCOMB, S2009ENE-1597 (Comunidad de Madrid), and ENE2008-06515-C04 and CSD2010-00011 (Ministerio de Ciencia e Innovación).

\section{References}

1. Clavin, P., Williams, F.A.: Effects of molecular difussion and of thermal expansion on the structure and dynamics of premixed flames in turbulent flows of large scale and low intensity. J. Fluid Mech. 116, 251-282 (1982) 
2. Matalon, M., Matkowsky, B.J.: Flames as gasdynamic discontinuities. J. Fluid Mech. 124, 239-259 (1982)

3. Clavin, P., Joulin, G.: Premixed flames in large scale and high intensity turbulent flow. J. Phys. Lett. 44, L1-L12 (1983)

4. Davis, S.G., Quinard, J., Searby, G.: Determination of Markstein numbers in counterflow premixed flames. Combust. Flame 130, 112-122 (2002)

5. Davis, S.G., Quinard, J., Searby, G.: Markstein numbers in counterflow, methane- and propaneair flames: a computational study. Combust. Flame 130,123-136 (2002)

6. Echekki, T., Mungal, M.G.: Flame speed measurements at the tip of a slot burner: Effects of flame curvature and hydrodynamic stretch. Proc. Combust. Inst. 23, 455-461 (1990)

7. Poinsot, T., Echekki, T., Mungal, M.G.: A study of the laminar flame tip and implications for premixed turbulent combustion. Combust. Sci. Technol. 81, 45-73 (1992)

8. Tseng, L.K., Ismail, M.A., Faeth, G.M.: Laminar burning velocities and Markstein numbers of hydrocarbon/air flames. Combust. Flame 95, 410-426 (1993)

9. Deshaies, B., Cambray, P.: The velocity of a premixed flame as a function of the flame stretch: An experimental study. Combust. Flame 82, 361-375 (1990)

10. Mungal, M.G., Lourenco, L.M., Krothapalli, A.: Instantaneous velocity measurements in laminar and turbulent premixed flames using on-line PIV. Combust. Sci. Technol. 106, 239-265 (1995)

11. Williams, F.A.: Combustion Theory, 2nd edn., Benjamin Cummings, Menlo Park, CA (1985)

12. Higuera, F.J.: Effects of the fresh gas velocity and thermal expansion on the structure of a Bunsen flame tip. Combust. Flame 157, 1586-1593 (2010)

13. Higuera, F.J.: Aerodynamics of a slender axisymmetric Bunsen flame with large gas expansion. Combust. Flame 156, 1063-1067 (2009)

14. Lewis, B., von Elbe, G.: Combustion, Flames and Explosions of Gases, chapter 5. Academic Press, New York (1961)

15. Clavin, P., Garcia-Ybarra, P.L.: The influence of the temperature dependence of difussivities on the dynamics of flame fronts. J. Méc. Théor. Appl. 2, 245-263 (1983)

16. Buckmaster, J.: A mathematical description of open and closed flame tips. Combust. Sci. Technol. 20, 33-40 (1979)

17. Buckmaster, J., Crowley, A.B.: The fluid mechanics of flame tips. J. Fluid Mech. 131, 341-361 (1983) 